\title{
Combining Classifiers through Triplet-Based Belief Functions
}

\author{
Yaxin $\mathrm{Bi}^{1}$, Shengli $\mathrm{Wu}^{1}$, Xuhui Shen ${ }^{2}$, and Pan Xiong ${ }^{2}$ \\ ${ }^{1}$ School of Computing and Mathematics, University of Ulster \\ Newtownabbey, Co. Antrim, BT37 0QB, UK \\ $\{\mathrm{y} . \mathrm{bi}, \mathrm{s} . \mathrm{wu} 1\} @ u l$ ster.ac.uk \\ ${ }^{2}$ Institute of Earthquake Science, China Earthquake Administration \\ Beijing, 100036 China
}

\begin{abstract}
Classifier outputs in the form of continuous values have often been combined using linear sum or stacking, but little is generally known about evidential reasoning methods for combining truncated lists of ordered decisions. In this paper we introduce a novel class-indifferent method for combining such a kind of classifier decisions. Specifically we model each output given by classifiers on new instances as a list of ranked decisions that is divided into 2 subsets of decisions, which are represented by triplet-based belief functions and then are combined using Dempster's rule of combination. We present a formalism for triplet-based belief functions and establish a range of general formulae for combining these beliefs in order to arrive at a consensus decision. In addition we carry out a comparative analysis with an alternative representation dichotomous belief functions on the UCI benchmark data. We also compare our combination method with the popular methods of stacking, boosting, linear sum and majority voting over the same benchmark data to demonstrate the advantage of our approach.
\end{abstract}

\section{Introduction}

The idea of ensemble learning or a committee approach is to learn and retain multiple classifiers and combine their decisions in some way to classify new instances [8]. Thus the key to the success of ensemble learning relies not only on a learning algorithm, but also on a combination function. In this work we focus on the latter task - developing an effective combination method. The design of a method for combining classifier decisions is a challenging task in constructing an effective ensemble and various methods have been developed in the past decades. Kuncheva in 12 roughly characterizes combination methods, based on the forms of classifier outputs, into two categories. In the first category, the combination of decisions is performed on single classes, such as majority voting and Bayesian probability, which have extensively been examined in the ensemble literature [9], 11] and [23].

The second category is concerned with the utilization of numeric scores (probabilities) corresponding to classes. One typical method, often called a 
class-aligned method, is based on the same classes from different classifiers in calculating the support for classes, regardless of what the support for the other classes is. This method includes meta-learning - stacking where combination functions are learnt from numeric values of classes [10] and [17, linear sum and order statistics [11, 21] and 24]. An alternative group of methods called classindifferent methods makes use of as much information as possible obtained from sets of classes in calculating the support for each class [12].

A class-aligned method and a class-indifferent method are both based on continuous values of classes in calculating the support for classes, but the latter takes impact from different classes into account in determining the support for classes that permits the presence of uncertainty information - as happens when an instance is classified into different classes by different classifiers. Several work related to class-indifferent methods utilizes single classes and sets of classes as described in [1] and [23]. However class-indifferent methods for combining decisions in the form of lists of ranked decisions have not been intensively studied and are poorly understood. In particular, little is known about evidential reasoning methods for combining truncated lists of ordered decisions.

In this study we propose a novel approach to modeling the process of combining classifiers in the Dempster-Shafer theory framework, which is built on our previous study for text categorization [4] and [5]. We model each output given by classifiers on new instances as a list of ranked decisions (classes) that is divided into 2 subsets of decisions along with the whole set of decisions which are represented by the structure called a triplet. In each triplet, the first subset contains a decision corresponding to the largest numeric score, the second subset corresponds to the second largest numeric value and the whole set of decisions represents the uncertainty in making the former two decisions in terms of $i g$ norance. In particular we extend our previous work by establishing the general formulae for combining triplets by Dempster's rule of combination and empirically and analytically examine the effect of different sizes of decision list on their accuracy for combining classifiers over the UCI benchmark data sets [7].

To evaluate the superiority of our method we have conducted a comparative analysis on the three representations of classifier decisions in the forms of tripletbased belief functions, dichotomous-based belief functions [3] and full list of probabilities, we also compare them with two state of the art methods: Stacking (multi-response linear regression) 17] and AdaBoost.M1 [16] along with majority voting and linear sum methods in combining individual (base) classifiers. By comparing the $t$-test results drawn from the various combinations of classifiers and the ranking statistics win/draw/loss, we demonstrate the properties and relative advantage of our method in combining classifiers.

\section{Modelling Classifier Outputs and Combination Methods}

In supervised machine learning, a learning algorithm is provided with training instances of the form $\left\{\left\langle d_{1}, c_{1}\right\rangle, \cdots,\left\langle d_{|D|}, c_{q}\right\rangle\right\}\left(d_{i} \in D, c_{i} \in C, 1 \leq q \leq|C|\right)$ for 
inducing some unknown function $f$ such that $f(d)=c$. $D$ is the space of attribute vectors and each vector $d_{i}$ is in the form $\left(w_{i_{1}}, \cdots, w_{i_{n}}\right)$ whose components are symbolic or numeric values; $C$ is a set of categorical classes and each class $c_{i}$ is in the form of class label. Given a set of training data, a learning algorithm is aimed at learning a function $\varphi-$ a classifier from the training data, where classifier $\varphi$ is an approximation to the unknown function $f$.

Given a new instance $d$, a classification task is to make the decision for $d$ using $\varphi$ about whether instance $d$ belongs to class $c_{i}$. Instead of single-class assignment, we denote such a process as a mapping:

$$
\varphi: D \rightarrow C \times[0,1]
$$

where $C \times[0,1]=\left\{\left(c_{i}, s_{i}\right)\left|c_{i} \in C, 0 \leq s_{i} \leq 1,1 \leq i \leq\right| C \mid\right\}, s_{i}$ is a numeric values that can be in different forms, such as a similarity score, a class-conditional probability (prior posterior probability) or other measures, depending on the types of learning algorithms. It represents the degree of support or confidence about the proposition of that instance $d$ is assigned to class $c_{i}$. The greater the value of class $c_{i}$, the greater the possibility of the instance belonging to that class. Without loss of generality, we denote the classifier output by $\varphi(d)=$ $\left\{s_{1}, \cdots, s_{|C|}\right\}-$ a general representation of classifier outputs. Given an ensemble of classifiers, $\varphi_{1}, \varphi_{2}, \cdots, \varphi_{M}$, all classifier outputs can be organized into a matrix called a decision profile as depicted in Fig. 1, the combination of classifier outputs can be carried out in different ways. One of the most commonly used combination

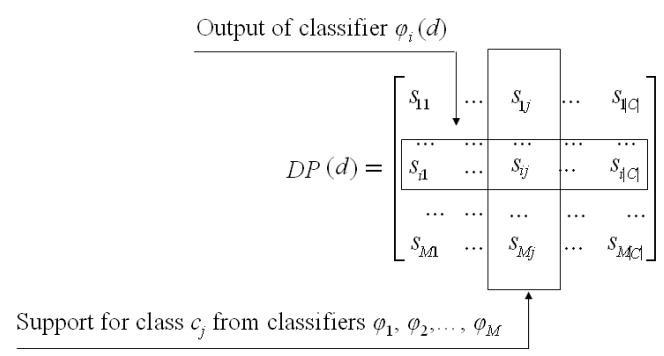

Fig. 1. A decision profile for instance $d$ generated by $\varphi_{1}(d), \varphi_{2}(d), \cdots, \varphi_{M}(d)$

methods is to calculate the support for class $c_{j}$ using only the $D P$ 's $j$ th column, i.e. $s_{1 j}, s_{2 j}, \cdots, s_{M j}$, regardless of what the support for the other classes is. We call such a methods a class-aligned method. Alternatively, the combination of classifier outputs can be performed on an entire decision profile or the selected information to constrain a class decision. We refer to this alternative group of methods as class-indifferent methods [12].

In our work, the concept of the class-indifferent methods is slightly different from the ones aforementioned in [1], 12] and [23]. We neither generate decision templates nor use an entire decision profile to compute the degrees of support for every class. Instead we select 2 classes from each $\varphi(d)$ according to their 
numeric values and restructure it into a new list composed of three subsets of $C$ which are represented by the novel evidence structure of triplet. In this way, a decision profile as illustrated in Fig. 1 will be restructured into a triplet decision profile where each column no longer corresponds to the same class. The degree of support for each class is computed through combining all triplets in a decision profile. We will detail our method in later sections.

\section{Dempster-Shafer (DS) Theory of Evidence}

In any exercise where decisions are to be combined, quantitative and qualitative pertinent information and knowledge often originate from different evidence sources, and they are often pervaded with uncertainty. In this study we seek a way to formalize such process in the context of ensembles for decision making with uncertainty. We briefly describe the DS theory of evidence below 19.

Definition 1. Let $\Theta$ be a finite nonempty set, and call it the frame of discernment. Let $[0,1]$ denote the interval of real numbers from zero to one, i.e. $[0,1]=\{x \mid 0 \leq x \leq 1\}$. A function $m: 2^{\Theta} \rightarrow[0,1]$ is called a belief mass function if it satisfies:

$$
\text { 1) } m(\emptyset)=0 ; \quad \text { 2) } \sum_{X \subseteq \Theta} m(X)=1 \text {. }
$$

A belief mass function is a basic probability assignment (bpa) to all subsets $X$ of $\Theta$. A subset $A$ of a frame $\Theta$ is called a focal element of a belief mass function $m$ over $\Theta$ if $m(A)>0$ and it is called a singleton if it contains only one element.

Definition 2. Let $m_{1}$ and $m_{2}$ be belief mass functions on the same frame $\Theta$, and for any subsets $A \subseteq \Theta$, the orthogonal sum $\oplus$ of two belief mass functions on $A$ is defined as

$$
m(A)=(1 / N) \sum_{X \cap Y=A} m_{1}(X) m_{2}(Y)
$$

where $N=1-\sum_{X \cap Y=\emptyset} m_{1}(X) m_{2}(Y)$ and $K=1 / N$ is called the normalization constant of the orthogonal sum $m_{1} \oplus m_{2}$. The orthogonal sum is a fundamental operation of evidential reasoning and it is often called Dempster's rule of combination. There are two conditions to ensure the orthogonal sum exists: 1) $N \neq 0 ; 2$ ) two belief mass functions must be independent of each other. We often allocate some mass to undeterministic status by means of ignorance.

It should be noted that the second condition above is a theoretical restriction on applying the orthogonal sum. In the case of classifier combination, it could be argued that the pieces of evidence derived from classifiers may not be entirely independent, but in this study we simply make an independence assumption on this. A recent discussion on this issue can be found in [2]. 


\section{Triplet-Based Belief Mass Function}

In this section we describe the development of a novel evidence structure - the triplet and its formulation.

Starting by analyzing the computational complexity of combining multiple pieces of evidence, we consider how a more efficient method for combining evidence can be established. Given $M$ pieces of evidence represented by Fig. 1, the computational complexity of combining these pieces of evidence using equation (2) is dominated by the number of elements in $C$ and the number of classifiers $M$. In the worst case, the time complexity of combining $M$ pieces of evidence is $O\left(|C|^{M-1}\right)$. One way of reducing the computational complexity is to reduce the number of pieces of evidence being combined, so that the combination of evidence is carried by a partition of the frame of discernment $C$, with less focal elements than $C$, but including possible answers to the proposition of interest. The partition can thus be used in place of $C$ when the computations of the orthogonal sum are carried out [18]. For example, a dichotomous structure can be used to partition the frame of discernment $C$ into two subsets $\vartheta_{1}$ and $\vartheta_{2}$, where there are a number of mass functions that represent evidence in favor of $\vartheta_{1}$ and against $\vartheta_{2}$, along with the lack of evidence - ignorance. It has been shown that Dempster's rule can be implemented in such a way that the number of computations increases only linearly with the number of elements in $C$ if the mass functions being combined are focused on the subsets where $\vartheta_{1}$ is singleton and $\vartheta_{2}$ is the complement of $\vartheta_{1}$, i.e., $O(|C|)$ 3].

The partitioning technique enables a large problem to be broken up into several smaller and more tractable problems. However, a fundamental issue in applying this technique is how to select elements that contain the possibly correct answers to the propositions corresponding to $C$.

An intuitive way is to select the element with the highest degree of confidence. Indeed, since the classifier outputs approximate class posteriori probabilities, selecting the maximum probability reduces to selecting the output that is the most 'certain' of the decisions. This could be justified from two perspectives. First, the probability assignments given in formula (1) give quantitative representation of judgments made by classifiers on the propositions; the greater their values, the more likely these decisions are correct. Thus selecting the maximum distinguishes the trivial decisions from the important ones. Second, the combination of decisions with the lower degrees of confidence may not contribute to the performance increase of combined classifiers, but only make the combination of classifiers decisions more complicated [21. The drawback of selecting the maximum, however, is that the combined performance can be reduced by a single dominant classifier that repeatedly provides high confidence values. Contenders with the higher values are always chosen as the final classification decisions, but some of these may not be correct.

To cope with the deficiency resulting from the maximal selection, we propose to take the second maximum decision into account in combining classifiers. Its inclusion not only provides valuable information contained in the discarded classes by the maximal selection for combining classifiers, but this also to some 
extent avoids the deterioration of the combined performance caused by the errors resulting from a single dominant classifier that repeatedly produces high confidence values. We propose a novel structure - a triplet - partitioning a list of decisions $\varphi(d)$ into three subsets below.

Definition 3. Let $C$ be a frame of discernment and let $\varphi(d)=\left\{s_{1}, \cdots, s_{|C|}\right\}$ be a list of scores, an application-specific belief mass function is defined as a mapping function, $m: 2^{C} \rightarrow[0,1]$, i.e. a bpa to $c_{i} \in C$ for $1 \leq i \leq|C|$ as follows:

$$
m\left(\left\{c_{i}\right\}\right)=\frac{s_{i}}{\sum_{j=1}^{|C|} s_{j}}
$$

where $1 \leq i \leq|C|$.

This mass function expresses the degrees of belief with regard to the choices of classes to which a given instance could belong. By equation (3), we can rewrite $\varphi(d)$ as $\varphi(d)=m\left(\left\{c_{1}\right\}\right), m\left(\left\{c_{2}\right\}\right), \cdots, m\left(\left\{c_{|C|}\right\}\right)$.

Definition 4. Let $\Theta$ be a frame of discernment and $\varphi(d)=\left\{m\left(\left\{x_{1}\right\}\right), m\left(\left\{x_{2}\right\}\right)\right.$, $\left.\ldots, m\left(\left\{x_{n}\right\}\right)\right\}$, where $|n| \geq 2$, an expression of the form $Y=\langle\{u\},\{v\}, C\rangle$ is defined as a triplet, where $\{u\},\{v\}$ are singletons, and $C$ is the whole set $\Theta$, and they satisfy

$$
m(\{u\})+m(\{v\})+m(C)=1
$$

To obtain triplet belief mass functions, we define a focusing operator and denote it by $m^{\sigma}$ as follows:

$$
\begin{gathered}
\{u\}=\arg \max \left\{m\left(\left\{x_{1}\right\}\right), m\left(\left\{x_{2}\right\}\right), \ldots, m\left(\left\{x_{n}\right\}\right)\right\} \\
\left.\{v\}=\arg \max \left\{m(\{x\}) \mid x \in\left\{x_{1}, \ldots, x_{n}\right\}-\{u\}\right)\right\} \\
C=\Theta, m^{\sigma}(\Theta)=1-m^{\sigma}(\{u\})+m^{\sigma}(\{v\})
\end{gathered}
$$

and each row in Fig. 1 is simply rewritten as formula (7) below.

$$
\varphi_{i}(d)=\left\{m^{\sigma}(\{u\}), m^{\sigma}(\{v\}), m^{\sigma}(C)\right\} 1 \leq i \leq M
$$

We refer to $m^{\sigma}$ as a triplet function or as a two-point mass function. For the sake of simplicity, $\varphi_{i}(d)=\{m(\{u\}), m(\{v\}), m(C)\}$. Following the same way, we divide $\varphi_{i}(d)$ into four subsets which is represented by three-point focuses called a quartet and define associated quartet belief mass functions. More details about quartets can be found in [6].

Suppose we are given two triplets $\left\langle\left\{x_{1}\right\},\left\{y_{1}\right\}, C\right\rangle$ and $\left\langle\left\{x_{2}\right\},\left\{y_{2}\right\}, C\right\rangle$ where $x_{i}, y_{i} \in C(i=1,2)$, and the associated triplet mass functions $m_{1}$ and $m_{2}$. The enumerative relations between any two pairs of focal elements $\left\{x_{1}\right\},\left\{y_{1}\right\}$ and $\left\{x_{2}\right\},\left\{y_{2}\right\}$ include: two focal points equal; one focal point equal; and totally different focal points. The general formulae for combining any number of triplet functions based on the three different cases are given below. 


\subsection{Two Focal Point Equal}

Considering a collection of triplet belief mass functions $m_{1}, \ldots, m_{l}$ that are defined on $\{x, y, \Theta\}, \ldots,\{x, y, \Theta\}$, by formula (2) - the orthogonal sum - we can derive the general formulae to combine these triplet functions:

$$
\begin{gathered}
m(\{x\})=K\left[\prod_{i=1}^{l}\left(m_{i}(\{x\})+m_{i}(\Theta)\right)-\prod_{i=1}^{l} m_{i}(\Theta)\right] \\
m(\{y\})=K\left[\prod_{i=1}^{l}\left(m_{i}(\{y\})+m_{i}(\Theta)\right)-\prod_{i=1}^{l} m_{i}(\Theta)\right] \\
m(\Theta)=K \prod_{i=1}^{l} m_{i}(\Theta) \\
K^{-1}=\prod_{i=1}^{l}\left(m_{i}(\{x\})+m_{i}(\Theta)\right) \\
+\prod_{i=1}^{l}\left(m_{i}(\{y\})+m_{i}(\Theta)\right)-\prod_{i=1}^{l} m_{i}(\Theta)
\end{gathered}
$$

\subsection{One Focal Point Equal}

Similarly we consider the case of any number of triplet belief mass functions $m_{1}, \ldots, m_{l}$ defined on $\left\{x, y_{1}, \Theta\right\}, \ldots,\left\{x, y_{l}, \Theta\right\}$, where $y_{1} \neq \ldots \neq y_{l}$, by repeatedly using formula (2) we then have

$$
\begin{gathered}
m(\{x\})=K\left(\prod_{i=1}^{l}\left(m_{i}\left(\left\{x_{i}\right\}\right)+m_{i}(\Theta)\right)-\prod_{i=1}^{l} m_{i}(\Theta)\right) \\
m\left(\left\{y_{i}\right\}\right)=K\left(m_{i}\left(\left\{y_{i}\right\}\right) \prod_{j=1, j \neq i}^{l} m_{j}(\Theta)\right) ; i \in\{1, \cdots, l\} \\
m(\Theta)=K \prod_{i=1}^{l} m_{i}(\Theta) \\
K^{-1}=\prod_{i=1}^{l}\left(m_{i}\left(\left\{x_{i}\right\}\right)+m_{i}(\Theta)\right)+ \\
m_{i}\left(\left\{y_{i}\right\}\right) \prod_{j=1, j \neq i}^{l} m_{j}(\Theta) ; i \in\{1, \cdots, l\}
\end{gathered}
$$




\subsection{Completely Different Focal Points}

Given a collection of triplet mass functions be $m_{1}, \ldots, m_{l}$ defined on $\left\{x_{1}, y_{1}, \Theta\right\}$, $\ldots,\left\{x_{l}, y_{l}, \Theta\right\}$, where $x_{i} \neq y_{j}, 1 \leq i, j \leq l$, by repeatedly using formula (2) to perform pairwise combination of any triplet functions, we then have

$$
\begin{gathered}
m\left(\left\{x_{i}\right\}\right)=K\left(\prod_{i \neq j}^{l}\left(m_{i}\left(\left\{x_{i}\right\}\right) m_{j}(\Theta)\right)\right. \\
m\left(\left\{y_{i}\right\}\right)=K\left(\prod_{i \neq j}^{l}\left(m_{i}\left(\left\{y_{i}\right\}\right) m_{j}(\Theta)\right)\right. \\
m(\Theta)=K\left(\prod_{i=1}^{l}\left(m_{i}(\Theta)\right)\right. \\
K^{-1}=\sum_{i=1}^{l} \prod_{i \neq j}^{l}\left(m_{i}\left(\left\{x_{i}\right\}\right) m_{j}(\Theta)+\right. \\
\sum_{i=1}^{l} \prod_{i \neq j}^{l}\left(m_{i}\left(\left\{y_{i}\right\}\right) m_{j}(\Theta)+\prod_{i=1}^{l}\left(m_{i}(\Theta)\right.\right.
\end{gathered}
$$

With a collection of triplet functions $m_{1}, m_{2}, \ldots, m_{n}$, simply it can be reorganized on the basis of one focus being equal, two focuses being equal, and none of focuses being equal as follows:

$$
m=\underbrace{m_{11} \oplus \ldots \oplus m_{1 l_{1}}}_{l_{1} \text { items }} \ldots \oplus \underbrace{m_{k 1} \oplus \ldots \oplus m_{k l_{k}}}_{l_{k} \text { items }}
$$

where $1 \leq k \leq 3 ; 0 \leq l 1, \ldots, l_{k}$ and $l_{1}+\ldots+l_{k}=n$, and $n$ is the number of items to be summed. For each $l_{i}$, we can use the formulae $(8)-(19)$ to calculate the combinations of $n$ triplet functions. In calculating Equation (20), it is assumed that at each step of pairwise calculation, the focusing operator will be applied to obtain a new triplet function, and a final decision to be made on a class assignment for an instance is based on the maximum selection of the belief given by Equation (20).

\section{Experimental Evaluation}

\subsection{Experimental Settings}

In our experiments, we used thirteen data sets downloaded from the UCI machine learning repository [7. All the selected data sets have at least three or more classes as required by the evidential structures. The details about these data sets can be found in Table 1 . 
Table 1. The general description about the datasets

\begin{tabular}{lccc}
\hline DATASET & \multicolumn{2}{c}{ INSTANCE No Classes AtTRIBUTE } \\
\hline ANNEAL & 798 & 6 & 38 \\
AUDIOLOGY & 200 & 23 & 69 \\
BALANCE & 625 & 3 & 4 \\
CAR & 1728 & 4 & 6 \\
GLASS & 214 & 7 & 9 \\
AUTOS & 205 & 6 & 25 \\
IRIS & 150 & 3 & 4 \\
LETTER & 20000 & 26 & 16 \\
HEART & 303 & 5 & 13 \\
SEGMENT & 1500 & 7 & 19 \\
SOYBEAN & 683 & 19 & 35 \\
WINE & 178 & 3 & 13 \\
ZOO & 101 & 7 & 17 \\
\hline
\end{tabular}

For base (individual) classifiers, we used thirteen learning algorithms which all are taken from the Waikato Environment for Knowledge Analysis (Weka) version 3.4 (see Table 2). These algorithms were simply chosen on the basis of their performance in three randomly picked data sets. For meta classifiers stacking, we chose the multi-response linear regression (MLR) and we also chose AdaBoostM1 to compare with our method. Parameters used for each algorithm were at the Weka default settings. The details about these algorithms can be found in 22$]$.

Table 2. The general description about thirteen learning algorithms

\begin{tabular}{clcl}
\hline No & Classifier & \multicolumn{2}{c}{ No Classifier } \\
\hline 0 & AOD & 1 & NAIVEBAYES \\
2 & SOM & 3 & IB1 \\
4 & IBK & 5 & KStar \\
6 & DeCisionStump & 7 & J48 \\
8 & RANDOMForest & 9 & DECISIONTABle \\
10 & JRIP & 11 & NNGE \\
12 & PART & & \\
\hline
\end{tabular}

The experiments were performed on a three partition scheme using a tenfold cross validation to avoid overfitting to some extent 1 . We divided the data sets into 10 mutually exclusive sets. For each fold, after the test set removal, the training set were further subdivided into $70 \%$ for a new training set and a $30 \%$ validation set. Apart from the evaluation of the performance of individual classifiers, the validation set is used to select the best combination of classifiers. The performance of the combinations of selected classifiers (the best ensembles of

\footnotetext{
${ }^{1}$ Two partition with a ten-fold cross validation was used for MLR and AdaBoostM1.
} 
classifiers) using DS, majority voting (MV) and linear sum (SUM) combination methods is evaluated on the testing set.

Eight groups of experiments are reported here, which were done individually and in combination across all the thirteen data sets. These include 1) assessing all the algorithms as shown in Table 2;2) combining the individual classifiers using DS, in which the outputs are represented by triplet functions and full list of probabilities (prior to transforming them to triplets); 3) combining the individual classifiers represented by the dichotomous functions using DS, where belief mass functions were defined on the basis of the performance of classifiers in terms of recognition, substitution and rejection rates $[23$; 4) combining the individual classifiers to construct the ensembles using MV, in which the individual outputs were in single classes; 5) combining the individual classifiers using SUM, in which the classifier outputs were in the form of full list of probabilities; 6) combining J48, NaiveBayes, MLR and KStar by MLR as used in [17]; 7) combining the best, the second best and the third best individual classifiers (SMO, IBk and NNge) by MLR; and 8) experimenting on AdaBoostM1 with SMO - the best individual classifier across the thirteen data sets.

To compare the classification accuracies between the individual classifiers and the combined classifiers across all the data sets, we employed the ranking statistics in terms of win/draw/loss record [14]. The win/draw/loss record presents three values, the number of data sets for which classifier $A$ obtained better, equal, or worse than classifier $B$ with respect to a classification accuracy. All collected classification accuracies were measured by the averaged $F$-measure 22 . A paired $t$-test across all these domains were also carried out to determine whether the differences between the base classifiers and combined classifiers are statistically significant at the 0.05 level.

\subsection{Experimental Results}

The ten experimental results are summarized in Tables 3 and 4, respectively, including all the classification accuracies of the best individual classifiers and the best combined classifiers. Table 3 presents the classification accuracies of the best individual classifiers and the best combined classifiers using DS under the four different structures. Table 4 gives the best combined classifiers using MV, SUM, boosting and stacking. In these tables, each cell represents either the accuracy of a best ensemble or the best individual classifier on the corresponding data set. If the difference between the best combined classifier and the best individual on the same data set is statistically significant, then the larger of the two is shown in bold.

The bottoms of Tables 3 and 4 provide summary statistics of comparing the performance of the best base classifiers with the best combined classifiers across the data sets. From these summaries, it can be observed that the accuracy of the combined classifiers based on the triplet structure using DS is better than the eight others on average. It has more wins to losses over the dichotomous, Fullist and the best combined classifiers using MV, SUM, boosting and stacking compared with the best individual classifiers. This finding is further supported 


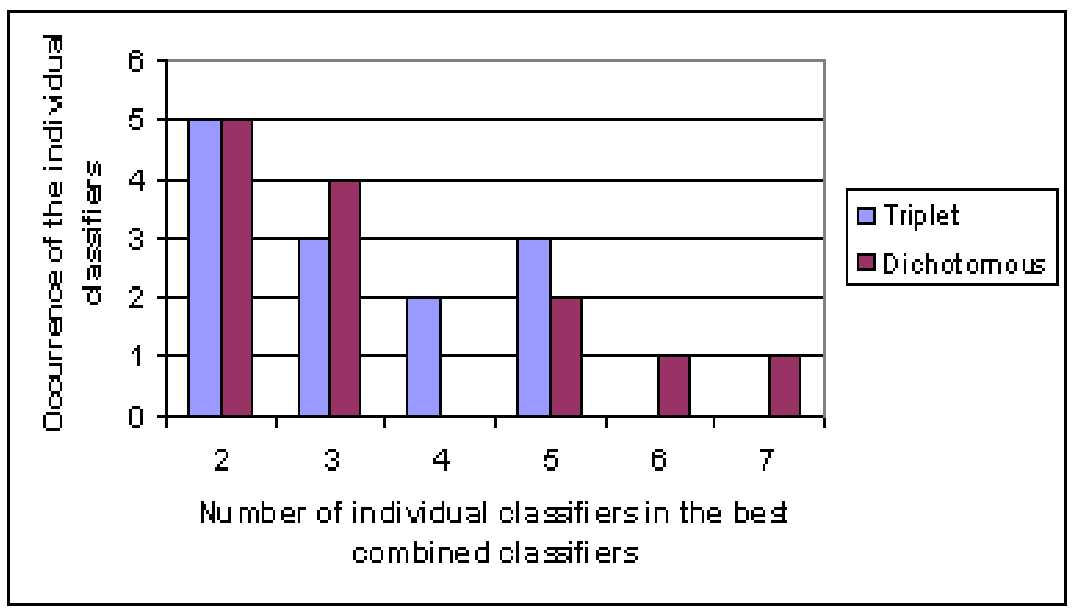

Fig. 2. Ensemble size: number of individual classifiers involved in the best combined classifiers across all the data sets with the evidential structures of triplet and dichotomous

Table 3. The classification accuracies of the best INDIVIDUAL classifier, best combined classifiers based on the different structures of TRIPLET, DICHOTOMOUS and FULLIST using DS over the thirteen data sets

\begin{tabular}{lcccc}
\hline DATASET & INDIVIDUAL TRIPLET & DICHOTOMS FULLIST \\
\hline ANNEAL & 80.23 & $\mathbf{8 1 . 5 7}$ & 80.68 & 69.88 \\
AUDIOLOGY & 48.67 & $\mathbf{5 7 . 4 4}$ & 51.97 & 49.32 \\
BALANCE & 65.67 & 63.17 & 65.67 & 21.68 \\
CAR & 89.62 & $\mathbf{9 4 . 2 9}$ & $\mathbf{9 1 . 9 2}$ & 90.03 \\
GLASS & 65.36 & 66.81 & 66.26 & 62.75 \\
AUTOS & 77.59 & 79.28 & 78.78 & 66.80 \\
IRIS & 95.33 & $\mathbf{9 6 . 6 7}$ & $\mathbf{9 6 . 6 7}$ & 60.71 \\
LETTER & 92.05 & $\mathbf{9 2 . 9 1}$ & $\mathbf{9 3 . 4 1}$ & 68.38 \\
CLEVELAND & 35.48 & 37.09 & 35.37 & 34.26 \\
SEGMENT & 96.69 & 97.35 & $\mathbf{9 7 . 7 4}$ & 88.40 \\
SOYBEAN & 95.89 & 96.88 & 96.85 & 91.11 \\
WiNE & 98.90 & $\mathbf{1 0 0 . 0 0}$ & 98.90 & 96.70 \\
ZOO & 90.62 & $\mathbf{9 3 . 6 1}$ & $\mathbf{9 3 . 6 1}$ & 64.39 \\
\hline \hline AVERAGE & 79.39 & 81.31 & 80.60 & 66.49 \\
Win/DRAW/LOSS & & $12 / 0 / 1$ & $10 / 2 / 1$ & $2 / 0 / 10$ \\
SigNIFICANT WIN & & 7 & 5 & 0 \\
\hline
\end{tabular}

by the statistically significant wins in which the triplet has two more wins than the dichotomous, three more wins than MV, four more wins than AdaBoost.M1, and seven more wins than Fullist and MLR.

Fig. 2 presents the sizes of the best combined classifiers across all the data sets. With the different structures, the construction of these ensembles involves 
Table 4. The classification accuracies of the best combined classifiers using MV, SUM, AdaBoost.M1 (BOOSTING corresponds to setting (8) in Section 5.1), and MLRs (STACK1,2 correspond to the settings (6) and (7) in Section 5.1) over the thirteen data sets

\begin{tabular}{|c|c|c|c|c|c|}
\hline DATASET & MV & SuM & Boosting & STACK1 & STACK2 \\
\hline ANNEAL & 81.14 & 80.51 & 77.35 & 72.77 & 75.34 \\
\hline AUDIOLOGY & 54.30 & 53.72 & 45.16 & 32.89 & 32.19 \\
\hline BALANCE & 62.72 & 63.17 & 93.17 & 62.73 & 68.49 \\
\hline CAR & 91.75 & 91.41 & 92.60 & 86.18 & 90.03 \\
\hline Glass & 66.69 & 66.40 & 65.97 & 58.41 & 57.77 \\
\hline Autos & 77.94 & 78.28 & 77.32 & 75.34 & 77.32 \\
\hline IRIS & 96.67 & 95.33 & 98.00 & 94.67 & 94.00 \\
\hline LETTER & 92.77 & 92.89 & 92.53 & 92.03 & 92.53 \\
\hline Cleveland & 34.37 & 37.64 & 31.91 & 35.13 & 31.87 \\
\hline SEGMENT & 96.55 & 97.68 & 96.57 & 96.59 & 95.85 \\
\hline SOYBEAN & 96.17 & 96.60 & 95.50 & 95.25 & 95.20 \\
\hline WINE & 98.97 & 99.42 & 98.38 & 98.90 & 98.32 \\
\hline Zoo & 93.61 & 93.61 & 89.43 & 82.57 & 83.64 \\
\hline AVERAGE & 80.28 & 80.51 & 81.07 & 75.65 & 76.35 \\
\hline WiN/DraW/LOSS & $10 / 0 / 3$ & $11 / 1 / 1$ & $5 / 0 / 8$ & $0 / 1 / 12$ & $3 / 0 / 10$ \\
\hline SignifiCANT Win & 4 & 3 & 3 & 0 & 0 \\
\hline
\end{tabular}

2-7 combinations of classifiers, and among them most of these combinations only involves two classifiers. This result is consistent with previous studies conducted in 4 ] and 20, and different from ones presented in [15] and [14] where their experiments showed that ensemble accuracy increased with ensemble size and the performance levels out with ensemble sizes of 10-25. Our experimental results uncover that due to the different way of constructing ensembles, the sizes of ensembles constructed by different learning algorithms working on a single data set is not necessarily the same as that constructed by single learning methods which manipulate different portion of features or instances of the data set.

\section{Discussion}

Perhaps one of the most important difference between the triplet-based classindifferent method from the single class label methods of AdaBoost.M1 and MV lies in the fact that it is motivated or built around the appealing intuition that the support contained in discarded classes - the second best decision - could help improve the combined performance of classifiers. More precisely the combined effect of two triplet classifiers will be affected by the first and second elements along with ignorance. Therefore the use of support from the other classes may play an important role in overcoming the single error produced by a classifier that repeatedly provides the high confidence values of classes as occurred in AdaBoost.M1 and MV. The empirical results show this property is appealing. 
Now we look at a theoretical justification on our claim. We state formally the conditions for the first or second decision in either of two triplets to become the best supported decision. Assume that two triplet functions $m_{1}$ and $m_{2}$ fall into the category where a pair of singletons $\left\{x_{1}\right\},\left\{y_{1}\right\}$ is equal to a pair of $\left\{x_{2}\right\},\left\{y_{2}\right\}$, i.e., $x=x_{1}=x_{2}$ and $y=y_{1}=y_{2}$ (see Section 4.1). By using formulae (2), we have the following inequality when $x$ is the best choice:

$$
\begin{gathered}
m_{1}(\{x\}) m_{2}(\{x\})+m_{1}(\{x\}) m_{2}(\Theta)+m_{1}(\Theta) m_{2}(\{x\})> \\
m_{1}(\{y\}) m_{2}(\{y\})+m_{1}(\{y\}) m_{2}(\Theta)+m_{1}(\Theta) m_{2}(\{y\})
\end{gathered}
$$

Substituting for $\Theta$ in formula (21) and rearranging it, we have the condition for the best support of $x$ below:

$$
m_{1}(\{x\})>1-\frac{\left[1-m_{1}(\{y\})\right]\left[1-m_{2}(\{y\})\right]}{\left[1-m_{2}(\{x\})\right]}
$$

Likewise we can derive the condition for $y$ being the best supported decision:

$$
m_{2}(\{y\})>1-\frac{\left[1-m_{1}(\{x\})\right]\left[1-m_{2}(\{x\})\right]}{\left[1-m_{1}(\{y\})\right]}
$$

We can obtain the conditions for the other two cases in two triplets in a similar manner.

The idea of MLR is to learn a single combination model for all the classes from the outputs of original classifiers using linear regression. It can be regarded to as a class-indifferent method. Our experimental results show that the performance of DS is significantly better than that of MLR and the combined classifiers by MV also outperform MLR that is consistent with the results on multi-class data presented in [17. The performance degradation of MLR might confirm its limited ability to determine decision boundaries for the multi-class data. Our experimental results also demonstrate this limitation could be improved to some extent by integrating the individual classifiers which are better performed.

With respect to the dichotomous structure, the drawback of that method is the way of measuring evidence, it ignores the fact that classifiers normally do not have the same performance on different classes, which could cause a deterioration in the performance of the combined classifiers.

Additionally compared with the performance of combined classifiers under the full list using DS, it can be observed that SUM is significantly better than DS. This finding suggests that to achieve the better performance of combining classifiers using DS, it is necessary to approximate the full list of probabilities (numeric scores) into some kind of structures, such as triplet. Inspecting the calculation process of Dempster's rule, it is not a surprise that the performance of combining classifiers in the form of triplets is better than that in a full list of decisions, because when a large number of decisions from each classifier are involved in combinations, it increases possibilities of conflict between two respective decisions - the larger the conflict, the poorer the performance of combined classifiers. This result is somehow consistent with the finding given in [13. 


\section{Conclusion}

We have presented a formalism for modelling classifier outputs in terms of triplets and the general formulae for combining base classifiers represented in the form of triplets which underpin our class-indifferent combination method. A wide range of experiments have been carried out over the thirteen UCI data sets. Our results show that the performance of the best combined classifiers is better than that of the best individuals at most of the data sets and the corresponding ensemble sizes are 2-7 where the combinations of 2 and 3 classifiers take $61.5 \%$ in the thirteen best ensembles. The comparative analysis among the structures of triplet, dichotomous and full list show that the triplet is the best one, and compared with the combinations methods of MV, SUM, MLR and AdaBoost.M1, DS is better than the others in combining the individual classifiers.

A decision rule defined on the basis of the DS theory of evidence is different from the majority voting principle. DS makes use of evidence accumulated from multiple classifiers in the process of classifier combination. It not only considers the majority agreement on the decisions received from classifiers, but it also incorporates the degrees of belief associated with those decisions into the decision making process. So it provides an effective means to reconcile decisions made by multiple classifiers.

\section{References}

1. Al-Ani, A., Deriche, M.: A new technique for combining multiple classifiers using the dempster-shafer theory of evidence. Journal of Artificial Intelligence Research 17, 333-361 (2002)

2. Altincay, H.: On the independence requirement in Dempster-Shafer theory for combining classifiers providing statistical evidence. Journal of Applied Intelligence 25, 73-90 (2006)

3. Barnett, J.A.: Combining opinions about the order of rule execution. In: AAAI, pp. 477-481 (1991)

4. Bi, Y., McClean, S.I., Anderson, T.: On combining multiple classifiers using an evidential approach. In: Proc of AAAI 2006, pp. 324-329 (2006)

5. Bi, Y., Bell, D., Wang, H., Guo, G., Guan, J.: Combining Multiple Classifiers for Text Categorization using Dempster's rule of combination. Journal of Applied Artificial Intelligence 21, 211-239 (2007)

6. Bi, Y., Wu, S., Guo, G.: Combining Prioritized Decisions in Classification. In: The International Conference of Modelling Decisions for Artificial Intelligence (2007)

7. Blake, C.L., Keogh, C.J.E.: Uci repository of machine learning databases, http://www.ics.uci.edu/ mlearn/MLRepository.html

8. Dietterich, T.: Ensemble methods in machine learning. In: Kittler, J., Roli, F. (eds.) MCS 2000. LNCS, vol. 1857, pp. 1-15. Springer, Heidelberg (2000)

9. Duin, R.P.W., Tax, D.M.J.: Experiments with classifier combining rules. In: Kittler, J., Roli, F. (eds.) Multiple Classifier Systems, pp. 16-29 (2000)

10. Dzeroski, S., Zenko, B.: Is combining classifiers with stacking better than selecting the best one? Machine Learning 54(3), 255-273 (2004)

11. Duin, R.P.W., Kittler, J., Hatef, M., Matas, J.: On combining classifiers. IEEE Trans. on pattern Analysis and Machine Intelligence 20(3), 226-239 (1998) 
12. Kuncheva, L.: Combining classifiers: Soft computing solutions. In: Pal, S.K., Pal, A. (eds.) Pattern Recognition: From Classical to Modern Approaches, pp. 427-451 (2001)

13. Kuncheva, L., Whitaker, C.J.: Measures of diversity in classifier ensembles. Machine Learning 51, 181-207 (2003)

14. Melville, P., Mooney, R.J.: Constructing diverse classifier ensembles using artificial training examples. In: Proc. of IJCAI 2003, pp. 405-510 (2003)

15. Opitz, D.: Feature selection for ensembles. In: Proc. of AAAI 1999, pp. 379-384. AAAI Press, Menlo Park (1999)

16. Schapire, R.E.: A brief introduction to boosting. In: Proc. of IJCAI 1999, pp. 1401-1406 (1999)

17. Seewald, A.K.: How to make stacking better and faster while also taking care of an unknown weakness. In: Proceedings of ICML 2002, pp. 554-561 (2002)

18. Shafer, G., Logan, R.: Implementing Dempster's Rule for Hierarchical Evidence. Artificial Intelligence 33(3), 271-298 (1987)

19. Shafer, G.: A Mathematical Theory of Evidence, 1st edn. Princeton University Press, Princeton (1976)

20. Tsymbal, A., Pechenizkiy, M., Cunningham, P.: Sequential Genetic Search for Ensemble Feature Selection. In: Proceedings of the Nineteenth International Joint Conference on Artificial Intelligence (IJCAI 2005) (2005)

21. Tumer, K., Robust, G.J.: On combining classifiers. Pattern Analysis and Applications 6(1), 41-46 (2002)

22. Witten, I.H., Frank, E.: Data Mining: Practical machine learning tools and techniques, 2nd edn. Morgan Kaufmann, San Francisco (2005)

23. Xu, L., Krzyzak, A., Suen, C.Y.: Several methods for combining multiple classifiers and their applications in handwritten character recognition. IEEE Trans. on System, Man and Cybernetics 2 (3), 418-435 (1992)

24. Ault, T., Yang, Y., Pierce, T.: Combining multiple learning strategies for effective cross validation. In: Proc of ICML 2000, pp. 1167-1182 (2000) 